\title{
Two-wave mixing in a broad-area semiconductor amplifier
}

\author{
Mingjun Chi, Søren Blaaberg Jensen \\ Optics and Plasma Research Department, Ris $\phi$ National Laboratory, DK-4000 Roskilde, Denmark \\ Jean-Pierre Huignard \\ Thales Research \& Technology, RD 128 91767, Palaiseau Cedex, France \\ Paul Michael Petersen \\ Optics and Plasma Research Department, Risф National Laboratory, DK-4000 Roskilde, Denmark \\ Mingjun.chi@risoe.dk, paul.michael.petersen@risoe.dk
}

\begin{abstract}
The two-wave mixing in the broad-area semiconductor amplifier was investigated, both theoretically and experimentally. In detail we investigated how the optical gain is affected by the presence of the twowave mixing interference grating. In the experimental setup we are able to turn on and off the interference pattern in the semiconductor amplifier. This arrangement allows us to determine the two-wave mixing gain. The coupled-wave equations of two-wave mixing were derived based on the Maxwell's wave equation and rate equation of the carrier density. The analytical solutions of the coupled-wave equations were obtained in the condition of small signal and the total intensity is far below the saturation intensity of the amplifier. The results show that when the amplifier is operated below transparency we obtain an increase in the optical gain, and when the amplifier is operated above transparency we obtain a decrease in the optical gain. The experimental results obtained in an $810 \mathrm{~nm}, 200 \mu \mathrm{m}$ wide GaAlAs amplifier show good agreement with the theory. A diffusion length of $2.0 \mu \mathrm{m}$ is determined from the experiment.
\end{abstract}

(C)2006 Optical Society of America

OCIS codes: (140.5960) Semiconductor lasers; (140.3280) Laser amplifiers; (190.7070) Twowave mixing

\section{References and Links}

1. H. Nakajima and R. Frey, "Collinear nearly degenerate four-wave mixing in intracavity amplifying media," IEEE J. Quantum Electron. 22, 1349-1354 (1986).

2. P. Kürz, R. Nagar, and T. Mukai, "Highly efficient phase conjugation using spatially nondegenerate fourwave mixing in a broad-area laser diode," Appl. Phys. Lett. 68, 1180-1182 (1996).

3. M. Lucente, G. M. Carter and J. G. Fujimoto, "Nonlinear mixing and phase conjugation in broad-area diode lasers," Appl. Phys. Lett. 53, 467-469 (1988).

4. M. Lucente, J. G: Fujimoto, and G. M. Carter, "Spatial and frequency dependence of four-wave mixing in broad-area diode lasers," Appl. Phys. Lett. 53, 1897-1899 (1988).

5. D. X. Zhu, S. Dubovitsky, W. H. Steier, K. Uppal, D. Tishinin, J. Burger, and P. D. Dapkus, "Noncollinear four-wave mixing in a broad area semiconductor optical amplifier," Appl. Phys. Lett. 70, 2082-2084 (1997).

6. P. M. Petersen, E. Samsøe, S. B. Jensen, and P. E. Andersen, "Guiding of laser modes based on self-pumped four-wave mixing in a semiconductor amplifier," Opt. Express 13, 3340-3347 (2005), http://www.opticsexpress.org.

7. P. Günter and J.-P. Huignard, eds., Photorefractive Materials and Their Applications I and II, (SpringerVerlag, Berlin, 1988, 1989).

8. A. Brignon and J.-P. Huignard, "Two-wave mixing in Nd:YAG by gain saturation," Opt. Lett. 18, 16391641 (1993).

9. G. P. Agrawal, "Four-wave mixing and phase conjugation in semiconductor laser media," Opt. Lett. 12, 260$262(1987)$ 
10. M. Chi, O. B. Jensen, J. Holm, C. Pedersen, P. E. Andersen, G. Erbert, B. Bumpf, and P. M. Petersen, "Tunable high-power narrow-linewidth semiconductor laser based on an external-cavity tapered amplifier," Opt. Express 13, 10589-10596 (2005), http://www.opticsexpress.org.

11. L. Goldberg, D. Mehuys, M. R. Surette, and D. C. Hall, "High-power, near-diffraction-limited large-area traveling-wave semiconductor amplifiers,” J. Quantum Electron. 29, 2028-2042 (1993).

12. J. R. Marciante and G. P. Agrawal, "Nonlinear mechanisms of filamentation in broad-area semiconductor lasers," J. Quantum Electron. 32, 590-596 (1996).

\section{Introduction}

Nonlinear four-wave mixing in narrow-stripe and broad-area semiconductor lasers is of interest as a method to obtain high phase conjugate reflectivity. ${ }^{1,2}$ The nonlinear wave mixing can also be used to measure carrier dynamics and gain behavior directly in the device, as well as for understanding device physics and application. ${ }^{3-5}$ Recently, we suggested that the gain and index grating created in broad-area semiconductor amplifier by four-wave mixing may be used to produce novel diode laser systems with better beam quality. ${ }^{6}$ Although two-wave mixing (TWM) has been intensively investigated in photorefractive materials, ${ }^{7}$ only few works was done in gain media. ${ }^{8}$ To our knowledge, this is the first time that the influence of the TWM grating on the optical gain is investigated in broad-area semiconductor amplifier.

Here, we present both the theoretical and experimental results of TWM in broad-area diode amplifier. The analytical solutions to the coupled-wave equations were obtained in the condition of small signal and the total intensity is far below the saturation intensity. The TWM experiment was carried out in an $810 \mathrm{~nm}, 200 \mu \mathrm{m}$ wide GaAlAs amplifier. The dependence of the TWM gain on the output power of the pump and angle between the two beams was measured. The experimental results show good agreement with the theory.

\section{Theory of two-wave mixing in broad-area semiconductor amplifier}

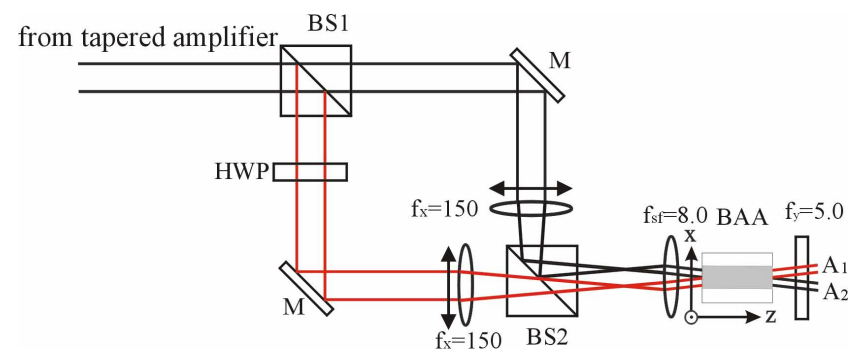

Fig. 1. Experimental set-up for the TWM in broad-area amplifier. M: mirror, BSs: beam splitter, HWP: half-wave plate, BAA: broad-area amplifier (the units are in $\mathrm{mm}$ ).

The TWM geometry is shown in Fig. 1, the pump beam of amplitude $A_{1}$ and signal beam of the amplitude $A_{2}$ are coupled into the broad-area amplifier. Both beams are at the same frequency $\omega$ and linearly polarized. The two beams interfere in the medium and a modulation of the carrier density in the active medium is caused, thus both a gain and a phase gratings are created. The nonlinear interaction in the gain media is governed by the wave equation:

$$
\nabla^{2} E-\frac{n^{2}}{c^{2}} \frac{\partial^{2} E}{\partial t^{2}}=\frac{1}{\varepsilon_{0} c^{2}} \frac{\partial^{2} P}{\partial t^{2}},
$$

where $n$ is the refractive index of the semiconductor material, and $c$ is the velocity of light in vacuum, and the $\varepsilon_{0}$ is the vacuum permittivity. The total electric field is given by: ${ }^{9}$

$$
E=A_{1} e^{i\left(K_{1} \cdot r-\omega t\right)}+A_{2} e^{i\left(K_{2} \cdot r-\omega t\right)},
$$

\#75857 - \$15.00 USD

(C) 2006 OSA
Received 6 October 2006; revised 20 November 2006; accepted 20 November 2006

11 December 2006 / Vol. 14, No. 25 / OPTICS EXPRESS 12374 
where $K_{1}$ and $K_{2}$ are the wave vectors of the pump and signal beam in the amplifier. $P$ is the induced polarization in the semiconductor amplifier. It is given by: ${ }^{9}$

$$
P=\varepsilon_{0} \chi(N) E
$$

where the susceptibility $\chi$ is given by: ${ }^{9}$

$$
\chi(N)=-\frac{n c}{\omega}(\beta+i) g(N)
$$

the quantity $\beta$ is the anti-guiding parameter accounting for the carrier-induced index change in semiconductor amplifier, and $g(N)$ is the material gain that is assumed to vary linearly with carrier density $N$, i.e. $g(N)=\Gamma a\left(N-N_{0}\right)$ where $a$ is the gain cross-section, $\Gamma$ is the confinement factor, and $N_{0}$ is the carrier density at transparency.

The carrier density $N$ is governed by the following rate equation ${ }^{6}$ :

$$
\frac{d N}{d t}=\frac{I}{q V}-\frac{N}{\tau}+D \nabla^{2} N-g(N) \frac{|E|^{2}}{\hbar \omega},
$$

where $I$ is the injected current, $q$ is the electron charge, $V$ is the active volume, $\tau$ is the spontaneous recombination lifetime, $D$ is the ambipolar diffusion constant, and $|E|^{2}=\left|A_{1}\right|^{2}+\left|A_{2}\right|^{2}$ the total intensity. In the TWM configuration the origin of the gain and index gratings is the modulation of the carrier density due to the interference between $A_{1}$ and $A_{2}$. Thus the carrier density that leads to the formation of the gratings may be written as:

$$
N=N_{B}+\Delta N \exp (-i K x)+\Delta N^{*} \exp (i K x),
$$

where $N_{\mathrm{B}}$ is the average carrier density, $\Delta N$ is the induced carrier modulation. $K=K_{2}-$ $K_{1}=4 \pi \sin (\theta / 2) / \lambda$ is the grating vector, where $\theta$ is the angle between the two beams and $\lambda$ is the wavelength. In the following perturbation analysis it is assumed that $\Delta N<<N_{\mathrm{B}}$. Inserting Eqs. (2) and (6) into Eq. (5), we find after some simple calculations that the average carrier density $N_{\mathrm{B}}$ and the carrier modulation $\Delta N$ are given by:

$$
\begin{aligned}
& N_{B}=\frac{I \tau / q V+N_{0}|E|^{2} / P_{s}}{1+|E|^{2} / P_{s}} \\
& \Delta N=-\frac{\left(N_{B}-N_{0}\right) A_{1} A_{2}^{*} / P_{s}}{1+D \tau K^{2}+|E|^{2} / P_{s}}
\end{aligned}
$$

where $P_{s}=(\hbar \omega) /(\Gamma a \tau)$ is the saturation intensity.

Inserting Eqs. (2) and (3) into Eq. (1), and using the obtained results of the average carrier density $N_{\mathrm{B}}$ and carrier modulation $\Delta N$, after some calculations, the coupled-wave equations for two-wave mixing are obtained: 


$$
\begin{aligned}
& \frac{\partial A_{1}}{\partial z}-i\left[-\frac{\alpha(\beta+i)}{1+|E|^{2} / P_{s}}\right]\left(1-\frac{\left|A_{2}\right|^{2} / P_{s}}{1+D \tau K^{2}+|E|^{2} / P_{s}}\right) A_{1}=0, \\
& \frac{\partial A_{2}}{\partial z}-i\left[-\frac{\alpha(\beta+i)}{1+|E|^{2} / P_{s}}\right]\left(1-\frac{\left|A_{1}\right|^{2} / P_{s}}{1+D \tau K^{2}+|E|^{2} / P_{s}}\right) A_{2}=0,
\end{aligned}
$$

where $\alpha=\Gamma a\left(I \tau / q V-N_{0}\right) / 2$ is the small-signal gain coefficient of the amplifier. The equations show that the coupling term between the two beams decreases the optical gain (above transparency) or absorption (below transparency) for both beams simultaneously. This is different to the situation in photorefractive materials, where one beam is amplified and the other is decreased at the same time.

To clarify this phenomenon, the relative position of the intensity pattern, the carrier density grating, the refractive index grating and the gain grating is shown in Fig. 2 when the amplifier is operated above the transparency. Because of the spatial hole-burning effect, the carrier density grating is $\pi$ out of phase with intensity pattern. Since the gain varies linearly with carrier density, the gain grating is also $\pi$ out of phase with the intensity pattern. The refractive index grating is in phase with the interference intensity pattern due to the antiguiding effect. The index grating has no contribution to the wave coupling, i.e. the energy exchange between two beams, when it is in phase or $\pi$ out of phase with the interference pattern. ${ }^{7}$ The gain grating will decrease the optical gain of both beams simultaneously because it is $\pi$ out of phase with the interference pattern. Reversely, the gain grating will decrease the absorption when the amplifier is operating below the transparency since the gain grating is in phase with the interference pattern.

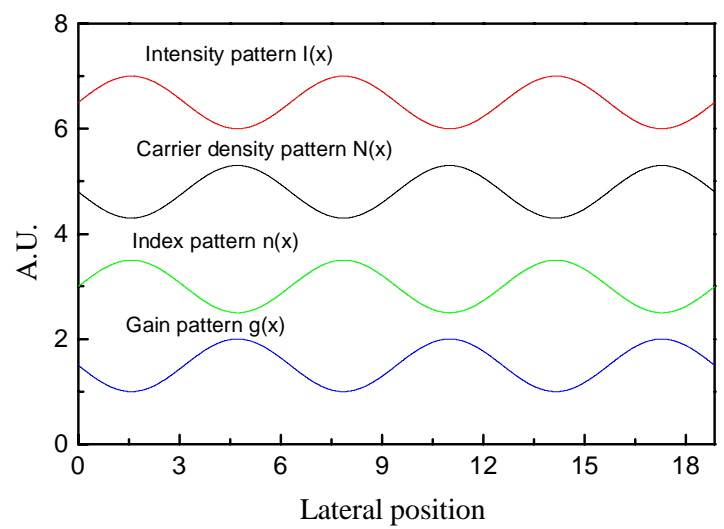

Fig. 2. The relative position of the interference pattern, the carrier density grating, the index and the gain grating formed in the BAA.

In the small signal approximation, and assume the total intensity of the beams is much less than the saturation intensity, i.e. $\left|A_{2}\right|^{2}<<\left|A_{1}\right|^{2}<<P_{s}$, the terms accounting for saturation in the denominators of the coupled-wave equations and the term accounting for the coupling in Eq. (9) are neglected. Thus the coupled-wave equations can be solved analytically. The solutions are: 


$$
\begin{aligned}
& A_{1}=A_{10} \exp [(1-i \beta) \alpha z], \\
& A_{2}=A_{20} \exp \left[(1-i \beta)\left(\alpha z-\gamma\left(e^{2 \alpha z}-1\right) / 2\right)\right],
\end{aligned}
$$

where $A_{10}$ and $A_{20}$ are the amplitudes of pump and signal beam at the front facet of the amplifier. $\gamma$ is a parameter defined as:

$$
\gamma=\frac{\left|A_{10}\right|^{2} / P_{s}}{1+D \tau K^{2}} .
$$

Define the TWM gain of the signal $g_{\mathrm{TWM}}$ as:

$$
g_{\text {TWM }}=\ln \left(\frac{\left|A_{2}\left(z_{0}\right)_{\text {coherent pump }}\right|^{2}}{\left|A_{2}\left(z_{0}\right)_{\text {noncoherent pump }}\right|^{2}}\right)=-\frac{\left|A_{1}\left(z_{0}\right)\right|^{2}-\left|A_{10}\right|^{2}}{\left(1+D \tau K^{2}\right) P_{s}},
$$

where $z_{0}$ is the length of the semiconductor amplifier. In the experiment, the coherent pump and the non-coherent pump is achieved by changing the polarization of the pump beam. Eq. (13) shows that the $g_{\text {TWM }}$ is negative when the amplifier is operated above the transparency, is positive when it is operated below the transparency, and is zero when it is operated at transparency. It agrees with the analyse above. Eq. (13) also shows that the $g_{\text {TwM }}$ decreases linearly with the output intensity (power) of the pump, and it decreases quickly when the angle between the two beams increases because the diffusion of carriers washes out the grating as the angle between the two beams increases. These analyses will be verified by experiments of TWM in a semiconductor amplifier below.

\section{Experiment}

The experimental set-up is shown in Fig. 1. The set-up is arranged like a Mach-Zehnder interferometer. The pump beam $A_{1}$ and the signal beam $A_{2}$ are derived from a tunable diode laser system based on a tapered amplifier. ${ }^{10}$ The wavelength used in the experiment is 813.5 $\mathrm{nm}$. We use the same method as in Ref. 11 to couple the two beams into the broad-area amplifier. In each arm, a combination of a cylindrical lens of $150 \mathrm{~mm}$ focal length and an aspherical lens of $8.0 \mathrm{~mm}$ focal length with a N.A. of 0.5 (this lens is shared by the two arms) is used as an afocal telescope to inject the two beams into the amplifier. The input coupling efficiency of this setup is around 50\%. The two cylindrical lenses can be translated in the arrow direction to vary the injection angle in the junction plane. After the amplifier, a cylindrical lens of $5.0 \mathrm{~mm}$ focal length is used to collimate the output beam in fast axis. A half-wave plate is inserted in the pump arm to change the polarization direction of pump. All the components are antireflection coated for the near infrared wavelength.

The broad-area amplifier is an $810 \mathrm{~nm}, 2 \mathrm{~mm}$ long and $200 \mu \mathrm{m}$ wide GaAlAs amplifier. It was grown by the Metallorganic Chemical Vapor Phase Deposition (MOCVD) technique on a GaAs substrate by Alcatel Thales III-V Lab. The structure contains a Large Optical Cavity (LOC), which has a thickness of approximately $1 \mu \mathrm{m}$, and which consists of a tensile-strained GaInP quantum well, two GaInP barriers and two AlGaInP claddings. Both facets of the amplifier are antireflection coated; the reflectivity is less than $0.1 \%$.

First, the dependence of the $g_{\mathrm{TWM}}$ on the output power of the pump is measured. The input powers of the pump and the signal measured before the aspherical lens are 21.0 and $4.1 \mathrm{~mW}$. The angle between the two beams is around $4^{\circ}$. The output power of the signal was measured at different injected current of the amplifier with a co-polarized pump (the polarization direction of both beams is perpendicular to the chip of the amplifier) and orthogonally- 
polarized pump. The output power of the pump was measured when it is coherent with the signal. The experimental results are shown in Fig. 3. It is clearly seen that the $g_{\text {TWM }}$ decreases linearly with the output pump power. Fitting the experimental data with Eq. (13), the two parameters: the input power of the pump $\left|A_{10}\right|^{2}$ and $1 /\left(1+D \tau K^{2}\right) P_{s}$ are obtained. The $\left|A_{10}\right|^{2}$ is round $9.1 \mathrm{~mW}$, corresponding to a coupling efficiency of $43 \%$; and using the results of $D \tau$ obtained later, the saturation power $P_{\mathrm{s}}$ is found to be around $220 \mathrm{~mW}$, which is much larger than the output pump power in this experiment. Using the value of $\left|A_{10}\right|^{2}$ and the Eq. (11), the optical gain of 1.7 is obtained for highest output power of the pump. The $g_{\text {TwM }}$ is about $5 \%$ of the optical gain.

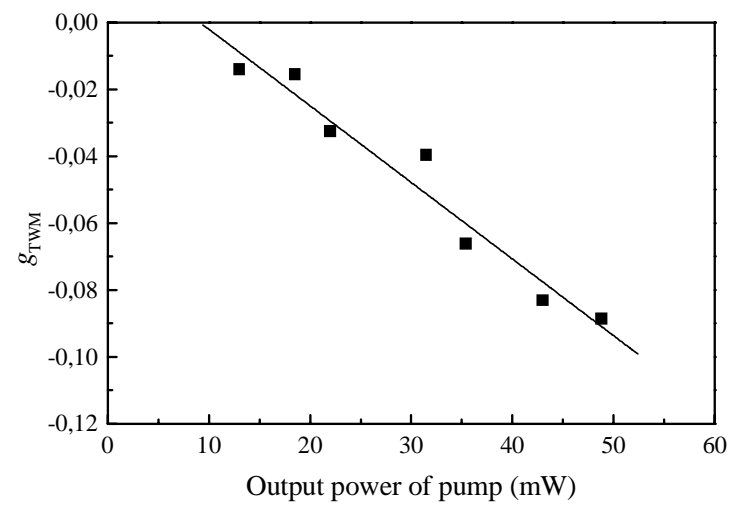

Fig. 3. The $g_{\text {TwM }}$ versus the output power of the pump. The squares are measured data; the line is the fitted result with Eq. (13).

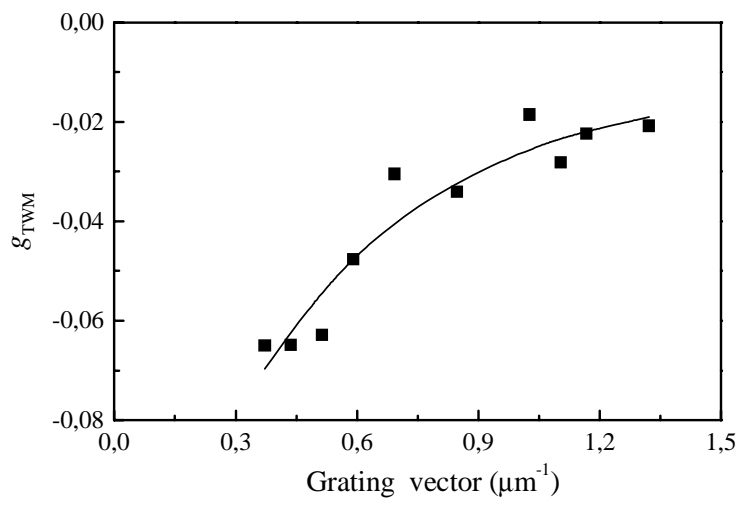

Fig. 4.The $g_{\text {TwM }}$ versus the grating vector in the BAA. The squares are measured data: the curve is the fitted result with Eq. (13).

The dependence of the $g_{\text {TWM }}$ on the grating vector is also measured by changing the angle between the two beams. The direction of the pump beam is fixed during the experiment; the angle is changed by changing the direction of the signal beam. The injected powers of the pump and the signal measured before the aspherical lens are 21.0 and $4.1 \mathrm{~mW}$; the output power of the pump is around $35 \mathrm{~mW}$. The experimental results are shown in Fig. 4. Fitting the experimental data with Eq. (13), D $\tau$ is obtained to be $4.1 \mu \mathrm{m}^{2}$, leading to a diffusion length 
$L=\sqrt{D \tau}$ of $2.0 \mu \mathrm{m}$. Assuming that $\tau$ is $5 \mathrm{~ns},{ }^{12} D$ is calculated to be $8.2 \mathrm{~cm}^{2} / \mathrm{s}$. This is in good agreement with the direct measured value of $9.5 \mathrm{~cm}^{2} / \mathrm{s}^{2}$ We should mention that the output power of pump beam is decreased a little when the polarization direction of it is changed from perpendicular to the chip to parallel to the ship. We do not know the reason of this decrease but the effect of this decrease on the measured $g_{\text {TWM }}$ is small.

To obtain the coupled-wave equations of TWM, three assumptions are made. Here we should discuss the validity of these assumptions in our experiment. The first is plane-wave assumption. In the experiment, since the two beams are coupled into the amplifier from an external laser, the mode of the two beams in the slow axis is determined by the external laser and the focusing optics. The two beams are nearly Gaussian beams in the slow axis, they are collimated by the aspherical lens and the width of the beams is around $140 \mu \mathrm{m}$. We believe the plane-wave assumption is a good approximation for these two beams in this direction. The wave guiding mode of field distribution in the fast axis does not affect the derivation of the equations. ${ }^{12}$ The second is the linear variation of material gain $g(N)$ on the carrier density. The transparent current of the amplifier used here is around 1.1 $\mathrm{A}$, and the highest current used in our experiment is $1.8 \mathrm{~A}$, according to Eq. (7), the carrier density $N_{\mathrm{B}}$ is calculated to be around $1.5 N_{0}$, not much higher than the transparent carrier density. The third assumption is the small population modulation in Eq. (6). With the injected current of $1.8 \mathrm{~A}$, according to Eq. (8), $|\Delta N|$ is calculated to be around $2 \%$ of $N_{0}\left(\approx 1.3 \%\right.$ of $\left.N_{\mathrm{B}}\right)$, it is much less than the carrier density $N_{\mathrm{B}}$. Therefore, we believe the assumptions made in the theory are valid in our experiment.

\section{Conclusion}

In conclusion, the TWM in broad-area semiconductor amplifier is investigated both experimentally and theoretically. The coupled-wave equations are derived and the analytical solutions are obtained when the intensity of the pump is much less than the saturation intensity of the amplifier, but much larger than that of the signal. The experimental results show good agreement with the theory. The validity of the theory is discussed.

\section{Acknowledgment}

The authors wish to acknowledge the financial support of the European Community through the FP-6 project WWW.BRIGHT.EU. Mingjun Chi wishes to acknowledge the Danish Research Agency under grant no. 26-04-0229.

\#75857 - \$15.00 USD

(C) 2006 OSA
Received 6 October 2006; revised 20 November 2006; accepted 20 November 2006

11 December 2006 / Vol. 14, No. 25 / OPTICS EXPRESS 12379 